\title{
FORMAS FARMACÊUTICAS DE LIBERAÇÃO MODIFICADA UTILIZADAS EM RUMINANTES: UMA REVISÃO
}

\author{
PRIETSCH, Rafael da Fonseca ${ }^{1}$; \\ PEREIRA, Rubens Alves ${ }^{1}$; \\ BRAUNER, Cássio Cassal ${ }^{1}$; \\ RABASSA, Viviane Rohrig ${ }^{1}$; \\ CORRÊA, Marcio Nunes ${ }^{1}$; \\ DEL PINO, Francisco Burkert ${ }^{1}$.
}

${ }^{1}$ Núcleo de Pesquisa, Ensino e Extensão em Pecuária (NUPEEC - www.ufpel.edu.br/nupeec), Universidade Federal de Pelotas, Faculdade de Veterinária, Campus Universitário, CEP 96010-900, Pelotas, RS, Brasil.

\section{RESUMO}

$\mathrm{N}$ a produção animal, a necessidade de inovação tecnológica para a promoção da saúde e do bem estar é impulsionada por vários fatores, dentre eles o de assegurar a sanidade animal e fornecer segurança aos consumidores. Estratégias inovadoras envolvendo sistemas de liberação modificada de fármacos têm surgido como alternativas promissoras para a terapêutica veterinária. No desenvolvimento destes sistemas de administração de fármacos, vários polímeros biodegradáveis têm sido utilizados por serem compostos facilmente assimiláveis pelo organismo do animal, além de melhorar os efeitos terapêuticos pela compatibilidade com as mais diversas classes farmacêuticas. $O$ sucesso destes sistemas está relacionado com a tecnologia de produção e com as características físico-químicas do polímero, garantindo vantagens como a redução do estresse animal e a diminuição dos riscos com a manipulação e a administração de fármacos. Entretanto, apesar destas vantagens, o preço elevado, a preocupação com resíduos, as questões regulatórias e os desafios na estabilidade da formulação, têm limitado o seu uso. Esta revisão tem por objetivo abordar formas farmacêuticas de liberação modificada de fármacos, utilizadas em ruminantes, buscando compreender as justificativas de seu desenvolvimento, os mecanismos tecnológicos envolvidos e as suas principais vantagens e desvantagens.

Palavras-chave: Sistemas de liberação. Fármacos veterinários. Tecnologia farmacêutica. 


\section{INTRODUÇÃO}

Na medicina veterinária, o medicamento é definido como toda a substância ou associação de substâncias químicas com propriedades preventivas ou curativas para doenças em animais; ou toda a substância ou associação de substâncias que possa ser administrada no animal com vista a restaurar, corrigir ou modificar funções fisiológicas, exercendo ação farmacológica, imunológica, metabólica ou de diagnóstico (BARBOSA, 2010).

Devido à importância dos medicamentos para a medicina veterinária, existe uma maior preocupação com o uso correto e racional, principalmente com os produtos destinados a animais de produção (FREITAS FILHO et al., 2011). O cuidado se deve principalmente ao excesso de dosagem que pode provocar o acúmulo de substâncias nos tecidos animais, aumentando o risco de contaminação humana. Durante o consumo de produtos de origem animal, por exemplo, a ingestão de alimentos com resíduos de antibióticos pode favorecer o desenvolvimento de resistência bacteriana a determinados fármacos, além de causar reações alérgicas e toxicidade (VAN DEN BOGAARD; STOBBERINGH, 2000).

Os medicamentos veterinários são tradicionalmente divididos de acordo com classes farmacológicas, cada uma abrangendo uma ampla variedade de propriedades químicas e físicas que muitas vezes dificultam a análise (identificação e quantificação) simultânea de diferentes substâncias em produtos de origem animal, o que coloca em risco a segurança alimentar humana devido à possibilidade de ingestão de produtos com resíduos de medicamentos (KAUFMANN et al., 2008).

Aliado a isso, outro fator que aumenta a preocupação supracitada, é o surgimento cada vez mais intenso de sistemas de administração modificada de fármacos, ou seja, das inovações farmacêuticas desenvolvidas com a promessa de otimizar a entrega do fármaco no local de ação, melhorar sua biodisponibilidade, diminuir a toxicidade, facilitar a administração, reduzindo o número de intervenções e favorecendo a eficiência dos tratamentos (FREITAS FILHO et al, 2011). Entretanto, apesar de todas as vantagens, alguns sistemas de liberação modificada de fármacos ao serem projetados para retardar ou prolongar a liberação das substâncias, dependendo da tecnologia empregada, acabam retardando também a eliminação das mesmas, pois promovem uma maior retenção no local de ação ou na 
corrente sanguínea, o que na prática exige um prolongado período de carência para o consumo de alimentos derivados (LYRA et al, 2007). Mesmo com o avanço constante no desenvolvimento de substâncias biodegradáveis, a segurança alimentar ainda é uma preocupação dos profissionais do setor de saúde quanto ao uso destes produtos inovadores (ROTHEN-WEINHOLD et al., 2000).

Diante disso, esta revisão tem por objetivo abordar formas farmacêuticas de liberação modificada utilizadas em ruminantes, buscando compreender as justificativas de seu desenvolvimento, seus mecanismos tecnológicos de funcionamento, suas principais características, além das possíveis desvantagens.

\section{FORMAS FARMACÊUTICAS DE LIBERAÇÃO MODIFICADA}

\section{Dispositivos Intraruminais}

Os ruminantes possuem várias características fisiológicas que fornecem importantes oportunidades para a administração de fármacos, como o estômago, composto por quatro câmaras: o rúmen, o retículo, o omaso e abomaso (CARDINAL; WITCHEY-LAKSHMANAN, 1992). O maior desses compartimentos é o rúmen, que pode ser visto como um grande tanque de fermentação, com pequenas aberturas para o esôfago e o omaso, representando uma região onde um dispositivo de administração controlada pode ser alojado, desde que possua um método adequado para evitar a regurgitação (ruminação) ou impedir que se mova através do trato gastrointestinal (CARDINAL, 1985). A fixação de dispositivos tem sido possível através da incorporação de substâncias de alta densidade, através da incorporação de sais de metais pesados ou por meio de dispositivos que expandem sua dimensão física garantindo a retenção no rúmen (WRIGHT, 1999). Outros, porém, têm sido desenvolvidos geralmente baseados em conceitos de partículas aprisionadas em reservatórios poliméricos, sistemas expansíveis ou dispositivos de distribuição osmótica (LAVY et al., 2006; RATHBONE; MARTINEZ, 2002; ROTHEN-WEINHOLD et al., 2000).

Neste sentido, muitas indústrias têm utilizado estas tecnologias para o lançamento de fármacos inovadores, especialmente anti-helmínticos. O Paratect Flex ${ }^{\oplus}$ Bolus (Pfizer Animal 
Health, New York, NY, EUA) é um exemplo de dispositivo projetado para profilaxia bovina com o anti-helmíntico morantel, a fim de impedir o estabelecimento de vermes gastrintestinais durante o pastejo por 90 dias (VANDAMME; ELLIS, 2004). É constituído por um cartão onde um trilaminado, uma matriz nuclear contendo uma mistura 50:50 de tartarato de morantel e acetato de vinil de etileno (EVA) são revestidos por um adesivo de EVA puro, que é impermeável ao fármaco. Após a entrada no rúmen, o adesivo dissolve-se e desenrola o dispositivo, aumentando suas dimensões e impedindo sua regurgitação (CARDINAL; WITCHEY-LAKSHMANAN, 1992). A substância é liberada pela difusão através de canais na matriz do núcleo poroso para as arestas não revestidas do dispositivo, ou por uma série de furos, através da matriz (ROTHEN-WEINHOLD et al., 2000). Para uma dada matriz de fármaco-polímero, a dose de fármaco liberada pode ser facilmente controlada por variação do número e diâmetro dos furos (BOETTNER et al., 1988).

Outro exemplo é o Ivomec ${ }^{\oplus}$ SR Bolus (Mountain View, CA, EUA), uma bomba osmótica que possui os compartimentos do agente osmótico e do fármaco separados por um êmbolo de cera, o qual foi concebido para administrar o anti-helmíntico ivermectina, para combater parasitas diretamente no rúmen, por um período de até 135 dias (BRAYDEN et al., 2010; MILLER et al., 2001; WRIGHT, 1999).

O Panacur ${ }^{\oplus}$ SR (Hoechst-Roussel, Intervet, Canada) é um dispositivo intraruminal de alta densidade para a liberação controlada de fembendazol, usado na profilaxia e no tratamento de infecções por nematódeos gastrintestinais em bovinos por até quatro meses (ROTHENWEINHOLD et al., 2000). O Captec ${ }^{\oplus}$ (Nufarm Pty, New Zealand) é uma cápsula intraruminal de libertação lenta de albendazol, que apresenta mais de $90 \%$ de eficácia no controle de parasitas por um período de até 90 dias (KLINK et al., 1998). O Rumensin ABC (Lilly, Indianapolis, IN, EUA) é um dispositivo intraruminal de liberação controlada de monensina, com eficácia de 100 dias, que utiliza asas que ficam dobradas durante a administração, mas posteriormente expandem, permitindo a sua retenção no rúmen (ELANCO, 2013). O Oros (Alza, Mountain View, CA, EUA) é uma mini bomba que libera hidromorfona em taxa controlada por até 24 horas (DROVER et al., 2002) e utiliza a tecnologia da bomba Push-Pull osmótica (Alza, Mountain View, CA, EUA). O Time Capsule (Ag Research, Hamilton, New 
Zealand) é uma tecnologia de liberação controlada através de bolus de liberação lenta de doses profiláticas de óxido de zinco, proporcionando até seis semanas de proteção contra eczema facial em ovinos e até cinco semanas em bovinos (PINTO et al., 2005).

\section{Dispositivos "bypass"}

São sistemas orais (normalmente multipartículados) de administração de substâncias destinados a passar intactos pelo rúmen, liberando as substâncias apenas no trato gastrintestinal inferior (CERVONI, 2013). Um exemplo de sistema bypass é a gordura protegida, que é uma fonte de ácidos graxos insaturados como o linoléico e o linolênico revestidos (GONÇALVES; DOMINGUES, 2007). Um exemplo comercial é o Megalac E (Arm\&Hammer Animal Nutrition, North Harrison Street, Princeton, EUA), que consiste em uma gordura granular revestida por sais de cálcio e óleo de palma que resistem à degradação ruminal (DOREAU; CHILLIARD, 1997). Outro exemplo de bypass é o Smartamine (Rhône-Poulenc Animal Nutrition, Antony, France), uma metionina encapsulada que é insolúvel no pH ruminal mas dissolve-se rapidamente no abomaso (PELL et al., 2000).

\section{Dispositivos Implantáveis}

Uma variedade de sistemas de entrega de liberação prolongada, que se destinam a servir como uma alternativa para a administração de hormônios de veículo líquido, por injeção, está disponível para o controle do ciclo estral, principalmente para bovinos e ovinos (KASTELIC et al., 1997). Um exemplo deste sistema é o Compudose ${ }^{\circledR}$, (Elancon Products Division of Eli Lilly Company, New Zealand), um implante polimérico de liberação prolongada que libera estradiol por um período de 84 a 100 dias, a fim de melhorar a taxa de crescimento e a eficiência alimentar em bovinos (DASH; CUDWORTHLI, 1998; PRESTON, 1999). Outro exemplo é o Ralgro (Schering-Plough Animal Health Corp., Union, N.J. EUA), um sistema de pellets implantáveis na orelha do animal com $12 \mathrm{mg}$ do hormônio zeranol, que tem uma eficácia de 90 a 100 dias em animais de qualquer idade. 
Outro dispositivo implantável é o SABER ${ }^{\mathrm{TM}}$ (Schering-Plough Animal Health, Mundelein, IL, EUA), um sistema de liberação que utiliza uma base de alta viscosidade para proporcionar a liberação controlada de progesterona e estradiol. Baseia-se em acetato isobutirato de sacarose, de baixo peso molecular, com muitas propriedades associadas com materiais poliméricos. Uma vez dentro do corpo, o solvente dissipa e um implante semi-sólido e biodegradável é formado (FLEURY et al., 1998, JOHNSON et al., 1999).

Por fim, o Atroban ${ }^{\circledast}$ (Schering-Plough Animal Health, Morris Avenue, EUA), um sistema auricular para administração de permetrina que atua por três meses, possui uma estrutura monolítica responsável por uma velocidade de liberação inicialmente elevada, que diminui ao longo do tempo. Porém, isso tem sido associado ao desenvolvimento de resistência ao inseticida, devido a uma prolongada exposição a doses subterapêuticas (BAGGOT, 1988).

\section{Dispositivos Transdérmicos}

Na medicina veterinária, a aplicação tópica de diversos fármacos tem sido considerada alternativa às vias de administração tradicionais (MILLS; CROSS, 2006). As formulações transdérmicas são alternativas mais eficientes para a veiculação de diversas classes de fármacos, pois superam obstáculos farmacocinéticos e farmacodinâmicos de diversos medicamentos quando utilizados por outras vias (BADRAN et al., 2009; TEICHMANN et al., 2007), além de prevenir a dor, a infecção e permitir o controle da liberação por longos períodos (JOSHI; RAJE, 2002; ROBERTS et al., 2002).

Apesar de proporcionar uma administração menos invasiva e permitir um melhor comprometimento do proprietário com a administração do fármaco (MAGNUSSON et al., 2001; ROBERTS et al., 2002), esta via depende de vários agentes farmacêuticos, além de limitar a administração de fármacos de alto peso molecular e de ter um atraso no início de ação farmacológica (GROND et al., 2000).

Um exemplo de sistema transdérmico é o Formoped (Pfizer Saúde Animal, São Paulo, Brasil), constituído de monometilol dimetil hidantoína e utilizado para podridão de cascos (footrot) e papilomatose bovina. É um potente bactericida e antisséptico, que não se altera 
na presença de matéria orgânica, destrói o tecido superficial de ulcerações, excrescências e infiltrações infecciosas no córion dos cascos e entre as unhas dos animais (PFIZER, 2013).

\section{Dispositivos Intravaginais}

A maioria dos sistemas intravaginais de liberação modificada de fármacos têm sido desenvolvida para a administração de hormônios naturais e sintéticos, tais como a progesterona, metil acetoxi progesterona, acetato de fluorogestona e benzoato de estradiol (BRAYDEN et al., 2010; RATHBONE; BRAYDEN, 2009). Estes sistemas possuem uma administração menos estressante, a liberação do fármaco termina no tempo pretendido, a vagina tolera facilmente, assegurando a retenção do dispositivo, entretanto deve-se ter cuidado com o risco do desenvolvimento de vaginites (ROTHEN-WEINHOLD et al., 2000).

Um exemplo é o $\mathrm{CIDR}^{\circledast}$ (Pfizer Saúde Animal, São Paulo, Brasil), um pessário intravaginal bovino, que libera progesterona gradativamente na mucosa vaginal (RUBIANES, 2000). É constituído de elastômero de silicone com progesterona, feito sobre um núcleo de nylon, tem o formato de " $\mathrm{T}$ " e possui abas flexíveis dobráveis formando uma haste para ser inserida na vagina com auxílio de um aplicador (BUNT et al., 1999). Também existe o CIDR-G ${ }^{\circledR}$, um dispositivo utilizado para a sincronização de estro em ovelhas e cabras (WHEATON et al., 1993), que é constituído por borracha de silicone em forma de espiral, banhada uniformemente com progesterona, contendo uma cápsula de gelatina dura ligada a superfície interna do espiral, e uma corda para auxiliar na remoção da cápsula (RATHBONE et al., 1999). Outro exemplo é o Primer ${ }^{\circledast}$ (Tecnopec, Saúde Animal. São Paulo, Brasil), o primeiro dispositivo intravaginal de progesterona desenvolvido no Brasil, baseado nas necessidades do rebanho nacional, contendo uma dose mais adequada (1 g) de progesterona, e usado em diferentes categorias animais como vacas e novilhas (BARROS, 2010). 


\section{Dispositivos Intramamários}

As formulações intramamárias são utilizadas para a prevenção ou tratamento de mastites clínicas e subclínicas em vacas leiteiras, doença que provoca uma redução na produção e qualidade do leite, resultando em perdas econômicas consideráveis (GEHRING, R.; SMITH, G. W., 2006). As estratégias terapêuticas contra a mastite envolvem a administração de formulações antimicrobianas de liberação imediata durante a lactação, ou formulações de ação prolongada durante o período seco (GRUET et al., 2001). Geralmente são adotadas formas líquidas e semissólidas, porém alguns parâmetros podem influenciar a bioequivalência, como o objetivo terapêutico (profilático ou para tratamento), o estágio de infecção (subclínica ou clínica) e se o animal está ou não em lactação (LAINESSE et al., 2012).

Algumas estratégias terapêuticas incluem o uso de imunomoduladores, tais como citocinas recombinantes que têm a capacidade de estimular leucócitos (ALLUWAIMI, 2004) e induzir um fluxo de neutrófilos para a glândula mamária quando aplicado no próprio lúmen do órgão (DALEY et al., 1993; SORDILLO et al., 1997). Outro medicamento disponível é a cloxacilina benzatina, um antibiótico $\beta$-lactâmico que é encapsulado em vetores nanoestruturados de quitosana, cujo o objetivo é aumentar a sua biodisponibilidade nas células infectadas da glândula mamária (ARAÚJO, 2009) e servir para a prevenção e tratamento da mastite (PEREZ et al., 1997). Outros exemplos de antibióticos usados são o Amoxi-Mast (Schering-Plough Animal Health Corp. Union, EUA) que contém amoxicilina, com largo espectro eficaz contra agentes causadores de mastite, que deve ser aplicado em três doses com intervalos de 12 horas, também o Spectramast LC (Pfizer, Saúde Animal, São Paulo, Brasil) que contém cloridrato de ceftiofur, é um intramamário potente para o tratamento de diferentes casos de mastite durante a lactação. Este antimicrobiano possui amplo espectro e alta eficácia contra bactérias gram-negativas e deve ser aplicado em todos os quartos afetados, repetindo-se o tratamento em 24 horas. Para terapias prolongadas, o tratamento diário com Spectramast LC ${ }^{\circledast}$ pode ser repetido por até oito dias consecutivos. Já o Masti-Clear ${ }^{\oplus}$ (G.C. Hanford, EUA) composto por penicilina G procaína, usado para o tratamento da mastite durante a lactação, é eficaz contra infecções causadas por Streptococcus agalactiae, Streptococcus dysgalactiae e Streptococcus uberis. Realiza-se uma 
aplicação de $10 \mathrm{~mL}$ do medicamento em cada quarto infectado, podendo-se repetir o tratamento em intervalos de 12 horas, até um total de três doses.

Além da utilização em diferentes formas farmacêuticas para a prevenção ou tratamento da mastite, a via intramamária também tem sido estudada para promover o aumento dos sólidos do leite, como por exemplo, através de infusões de $10 \mathrm{mg}$ de insulina liofilizada e LR3-IGF-I (fator de crescimento semelhante à insulina 1), realizadas com o objetivo de aumentar a proteína do leite (MACKLE et al., 2000).

\section{Dispositivos Intramusculares}

A via intramuscular é frequentemente utilizada por possuir uma absorção mais uniforme, permitindo a administração de substâncias aquosas ou oleosas, suspensões, além de ser de fácil aplicação em zonas distintas do animal (REEVES, 2007). Em grandes animais, além do músculo da coxa, é comum a aplicação nos músculos do pescoço, para evitar manchas na carcaça (SMITH et al., 1998), sendo realizada diretamente no músculo esquelético subjacente à camada subcutânea (MEDLICOTT et al., 2004). Entretanto, a aplicação intramuscular pode causar dor e aparecimento de lesões musculares pela aplicação de substâncias irritantes ou com pH distante da neutralidade, desencadeando processos infamatórios (SPINOSA et al., 1999). Além do mais, o acúmulo de resíduos do medicamento no músculo, principalmente em gado de corte, pode comprometer a segurança alimentar (REEVES, 2007). A deposição destes resíduos está relacionada a fatores como volume utilizado na injeção e local anatômico da administração. Em bezerros, há menor acúmulo de resíduos nos músculos do pescoço comparado com os músculos do glúteo (GEORGE et al., 1997).

Um exemplo de dispositivo intramuscular é o MAD-4 (Rio de Janeiro, Argentina), que possui progesterona para administração em vacas com o objetivo de preparar o útero para a gestação (ROCHA et al., 2011). Alguns estudos farmacocinéticos atuais demonstram a importância desta via para a medicina veterinária de grandes animais quando comparada à via intravenosa (ARESKOG et al., 2012; HORWITZ et al., 2011; KUMAR et al., 2012). 


\section{Dispositivos Subcutâneos}

A via de administração subcutânea é uma alternativa eficiente para os sistemas de liberação controlada. A adição de agentes que aumentam a viscosidade de soluções tem sido utilizada para prolongar o tempo de absorção, diminuindo a difusão no local da aplicação (MEDLICOTT, 2004). Esta via tem como desvantagem o risco de produzir sensibilização, dor e necrose, quando usadas substâncias irritantes (SPINOSA et al., 1999).

Um estudo utilizando as substâncias ivermectina (Ivomec ${ }^{\circledR}$, Merial Saúde Animal, Brasil) e amoxicilina (Clamoxyl ${ }^{\oplus}$, Laboratórios Pfizer, Brasil), demonstrou que através da combinação desses fármacos com veículos hidrofóbicos, é possível prolongar a ação dos mesmos, sendo importante em casos de grandes intervalos posológicos. (ROTHEN-WEINHOLD et al., 2000).

Implantes subcutâneos de orelha, como Syncro-Mate-B ${ }^{\circledast}$ e Syncro-Atec ${ }^{\oplus}$ (CEVA, Libourne, França) foram desenvolvidos contendo o hormônio norgestomet para uso em bovinos. Outro exemplo semelhante é Melovine (CEVA, Portugal), implante de orelha que contém melatonina para ovinos e caprinos, liberada em doses progressivas por um período de três a quatro meses, semelhantes às secretadas naturalmente durante a noite (VALENTIM et al., 2006). O Crestar (Akzo Nobel Ltda. - Divisão Intervet, Brasil) é colocado subcutaneamente na orelha de bovinos e retirado depois de nove dias, associado à injeção de $3 \mathrm{mg}$ de norgestomet e $5 \mathrm{mg}$ de valerato de estradiol, aplicada no momento da colocação do implante (TREGASKES et al., 1994).

O Atrigel ${ }^{\oplus}$ (Atrix Laboratories, Fort Collins, Colorado, EUA) foi desenvolvido para a entrega de antígenos complexos, tal como o vírus da pseudo-raiva (PRV), inativado em porcos (BOWERSOCK; MARTIN, 1999). Produzido através da mistura de polímeros (poliláctidos e poliglicólidos) com o solvente e a vacina, este sistema de liberação aumenta os níveis de anticorpos no soro por mais de 90 dias.

Os sistemas subcutâneos de liberação controlada também têm sido utilizados para administração de vitamina B12 em ovinos (SMART Shot ${ }^{\circledR} B_{12}$, New Zealand). Cada $\mathrm{mL}$ da formulação contém $6 \mathrm{mg}$ de vitamina $B_{12}$, hidroxocobalamina, encapsulada num copolímero láctido/glicólido que controla a liberação (GRACE et al., 2003). A sua administração em 
cordeiros garante doses de 3, 4, 5 ou $6 \mathrm{mg}$ de vitamina $B_{12}$, proporcionando maiores ganhos de peso.

\section{Dispositivos Tópicos}

O principal representante das formulações tópicas para animais de produção é o pour-on, uma emulsão que controla a liberação do fármaco e é derramada no dorso do animal. Para ser eficaz deve ter uma penetração suficiente na pele para permitir a absorção sistêmica e ao mesmo tempo evitar a remoção do fármaco em condições ambientais como chuva, sol e frio (ANDRESS et al., 2000; STROMBERG et al., 1999). O pour-on é um método geralmente utilizado para a administração de ivermectina em bovinos, podendo ter ação local ou sistêmica, porém pode ter diferentes efeitos numa pele íntegra, em relação à pele alterada. Um exemplo de pour-on é o Acatak (Novartis, Saúde Animal Ltda., Brasil) que contém o princípio ativo fluazuron, utilizado para o controle do carrapato em bovinos, protegendo o animal por um período de 8 a 12 semanas (BULL et al., 1996). Outro exemplo é o Supramec pour-on (Shering-Plough, Coopers, São Paulo, Brasil) que contém ivermectina e é utilizado para o tratamento e controle de parasitas internos e externos em bovinos. Já o Dectomax pour-on (Zoetis Inc., EUA) é uma solução de doramectin, um parasiticida de amplo espectro altamente eficaz para bovinos (LANUSSE et al., 1997).

\section{Polímeros Biodegradáveis}

Nos últimos anos, os sistemas biodegradáveis de administração de fármacos veterinários, tais como microesferas e implantes, foram testados na área de controle do estro (MATSCHKEET et al., 2002), na promoção de crescimento (ROTHEN-WEINHOLD et al., 2000) e no controle de ectoparasitas (MILLER, 2000). As vantagens de sua utilização é que os sistemas de entrega com base em polímeros biodegradáveis não exigem a remoção após o tratamento devido à sua degradação em compostos que podem ser facilmente assimilados pelo organismo (WINZENBURGN et al., 2004). No entanto, atualmente poucos sistemas biodegradáveis de administração de fármacos estão disponíveis comercialmente para uso veterinário, principalmente devido ao preço elevado, possibilidade de ocorrer ruptura da 
membrana e causar uma liberação brusca do fármaco, preocupação com resíduos, seguido por considerações regulatórias e desafios na estabilidade da formulação, o que têm limitado até mesmo seu desenvolvimento (WINZENBURG et al., 2004).

\section{Nanopartículas}

As nanopartículas farmacêuticas são sistemas transportadores de fármacos formados por polímeros biodegradáveis com diâmetro inferior a 1 m (MARTIN; KOHLI, 2003). Juntamente com os lipossomas, as nanoesferas e as nanocápsulas compreendem os principais sistemas nanoestruturados, sendo que as duas últimas diferem entre si quanto à composição e organização estrutural (SCHAFFAZICK et al., 2003). As principais vantagens do emprego da nanotecnologia é o uso racional de medicamentos, uma vez que tanto o número de doses quanto a concentração podem ser reduzidos durante o tratamento, assim como a inovação de bases farmacêuticas já desgastadas, uso de novas vias de aplicação, menor estresse na administração do medicamento, redução da toxicidade e dos efeitos adversos, uso de novas moléculas e novas formulações na terapêutica animal (BRANDÃO et al., 2011).

Até o momento foram propostas diferentes estratégias nanotecnológicas para a administração de fármacos, as quais possuem entre outras características, a capacidade de proteger um fármaco contra a degradação, melhorar a sua absorção facilitando a difusão através do epitélio, alterar o perfil farmacocinético e de distribuição do fármaco e/ou melhorar a penetração e distribuição intracelular (COUVREUR; VAUTHIER, 2006). A via injetável e implantes subcutâneos são alguns exemplos de sistemas que utilizam fármacos sob a forma de micro ou nanopartículas, tendo assim uma melhor eficácia e efeitos colaterais diminuídos (REVERCHON, 1999). Já os lipossomas são adequados para administração tópica, intravenosa e intramuscular (BAKKER-WOUDENBERG et al., 2005; DAMS et al., 2000). Algumas formulações de lipossomas já estão disponíveis no mercado, além de estudos sobre suas aplicações em pequenos animais (HAFEMAN et al., 2010; KLEITER et al., 2010), em bovinos (LYNN et al., 2004) e ovinos (USUI et al., 2003) estarem acontecendo em todo o mundo. 
De uma forma geral, a aplicabilidade da nanotecnologia na medicina veterinária, principalmente de animais de produção, ainda é insignificante quando comparada à medicina humana, principalmente devido à alta tecnologia necessária, a qual requer altos investimentos.

\section{Micropartículas}

Os sistemas de micropartículas têm sido investigados para proporcionar a liberação sustentada de fármacos durante períodos de semanas até um ano ou mais (SCHAFFAZICK et al., 2003). Vários métodos de preparação são utilizados, incluindo diferentes solventes orgânicos que têm sido empregados na formulação de microesferas de progesterona e estradiol para o controle de cio e ovulação (BURNS et al., 1994). Estes sistemas melhoram a proteção do fármaco, podem promover muco adesão e o fracionamento da dose, contribuindo para melhorar a biodisponibilidade das substâncias (LOPEZ-DONAIRE et al., 2010; SHAHANI et al., 2010). Entretanto, apresentam desvantagens como solvente residual na dispersão final e o fato das dispersões serem muito diluídas, devido à limitada solubilidade do lipídeo no solvente orgânico (WISSING et al., 2004). Além disso, alguns fármacos possuem baixa capacidade de encapsulação, podendo causar a liberação do fármaco até mesmo durante a estocagem (SATO; UENO, 2005).

Um promissor exemplo é a microencapsulação de antígenos com polímeros biodegradáveis, tecnologia usada para potencializar as características imunogênicas (HILBERT et al., 1999). Sistemas de liberação controlada permitem a liberação contínua de antígenos encapsulados, especificamente no local de ação, protegendo contra uma possível degradação, garante maior comodidade ao paciente, devido ao número menor de aplicações e uma dose antigênica mais efetiva (LIMA; RODRIGUES-JUNIOR, 1999; PEREZ et al., 2002). Outros estudos relatam a eficácia, em longo prazo, de micropartículas de ivermectina para bovinos e caprinos no controle de carrapatos utilizando polímeros biodegradáveis (MILLER et al., 1998). 


\section{CONCLUSÃO}

Existem inúmeras formas farmacêuticas de liberação modificada disponíveis na medicina veterinária de grandes animais, as quais utilizam tanto tecnologias simples, quanto ferramentas mais complexas e inovadoras desenvolvidas pela indústria farmacêutica. A intensa investigação científica empregada na medicina veterinária tem contribuído para uma maior compreensão dos mecanismos de ação das substâncias terapêuticas e fornecido melhores subsídios para o desenvolvimento de formulações cada vez mais específicas para determinadas espécies e/ou para determinadas enfermidades.

Os sistemas de liberação de fármacos variam conforme a tecnologia empregada, o que pode influenciar diretamente no sucesso terapêutico em todas as situações clínicas. Portanto, o conhecimento do mecanismo de funcionamento das formulações de liberação modificada, bem como de suas possíveis desvantagens, pode permitir ao profissional veterinário fazer avaliações racionais que possibilitem a adequada escolha do produto farmacêutico, bem como a melhor forma de otimizar os seus resultados terapêuticos. 


\section{MODIFIED RELEASE DOSAGE FORMS USED IN RUMINANTES: A REVIEW}

\section{ABSTRACT}

I

$\mathrm{n}$ animal production, the need for technological innovation to promote the health and welfare is driven by several factors among them to ensure animal health and provide safety to consumers. Innovative strategies involving with modified release of drugs emerge as promising alternatives to veterinary therapeutic. In the development of these drug delivery systems, various biodegradable polymers have been used because are easily assimilated by the animal organism and can improve the therapeutic effects by having compatibility with several drugs classes. The success of these systems is related to the production technology and the physiochemical characteristics of the polymer, providing advantages such as reducing animal stress and of the risks during the handling and administration of drugs. However, despite these advantages the high price, the concern with waste, regulatory issues and challenges in the formulation stability, have limited its use. The objective of this review is present modified release dosage forms used in ruminants, trying to understand the reasons for its development, the technological mechanisms involved, well as their main advantages and disadvantages.

Keywords: Release systems. Veterinary drugs. Pharmaceutical technology.

\section{FORMAS FARMACÉUTICAS DE LIBERACIÓN MODIFICADA UTILIZADAS EN RUMIANTES: UNA REVISIÓN}

\section{RESUMEN}

$\mathrm{E}$ n la producción animal, la necesidad de innovación tecnológica para promoción de la salud y del bienestar es impulsada por varios factores, dentro de estos, el de asegurar la sanidad animal y ofrecer seguridad a los consumidores. Estrategias innovadoras envolviendo sistemas de liberación modificada de fármacos han surgido como alternativas promisorias para la terapéutica veterinaria. En el desenvolvimiento de estos sistemas de administración de fármacos, varios polímeros biodegradables han sido utilizados por ser compuestos fácilmente asimilables por el organismo del animal, además de mejorar los efectos terapéuticos por la compatibilidad con las más diversas formas farmacéuticas. El éxito de estos sistemas está relacionado con la tecnología de producción y con las características físico-químicas del polímero, garantizando ventajas como la reducción del estrés animal, disminución de los riesgos con la manipulación y administración de fármacos. Sin embargo, a pesar de estas ventajas, el costo elevado, la preocupación con los residuos, las cuestiones regulatorias y los desafíos en la estabilidad de la formulación, han limitado su uso. Esta revisión tiene por objetivo abordar formas farmacéuticas de liberación modificada de fármacos utilizadas en rumiantes, buscando comprender las justificativas de su 
desenvolvimiento, los mecanismos tecnológicos envueltos y sus principales ventajas y desventajas.

Palabras clave: Sistemas de liberación. Fármacos veterinarios. Tecnología farmacéutica.

\section{REFERÊNCIAS}

ALLUWAIMI, A. M. The cytokines of bovine mammary gland: prospects for diagnosis and therapy. Research in Veterinary Science, v. 77, n. 3, p. 211-222, 2004.

ANDRESS, E. R.; DE ROUEN, S. M.; FOIL, L. D. Efficacy of doramectin $0.5 \%$ w/v pour-on for control of the horn fly, Haematobia irritans, Veterinary Parasitology, v. 90, n. 4, p. 327-331, 2000.

ARAÚJO, R. S. Desenvolvimento, caracterização e liofilização de nanoparticulas e encapsulamento de antibiótico de uso veterinário. Ouro Preto: UFOP, 2009. 131f. Dissertação (Mestrado em Ciências Farmacêuticas), Escola de Farmácia da Universidade Federal de Ouro Preto, Ouro Preto, 2009.

ARESKOG, M.; HIMMELSTJERNA, G. S.; ALVINERIE, M.; et al. Dexamethasone treatment intereferes with the pharmacokinetics of ivermectin in young cattle. Veterinary

Parasitology, v. 190, n 3-4, p.482-488, 2012.

BADRAN, M. M.; KUNTSCHE, J.; FAHR, A. Skin penetration enhancement by a microneedle device (Dermaroller ${ }^{\circledast}$ ) in vitro: Dependency on needle size and applied formulation.

European Journal of Pharmaceutical Sciences, v. 36, p. 511-523, 2009.

BAGGOT, J. D. Veterinary drug formulations for animal health care: an overview. Journal of Controlled Release, n. 8, p. 5-13, 1988.

BAKKER-WOUDENBERG, I. A.; SCHIFFELERS, R. M.; STORM, G. et al. Long-circulating sterically stabilized liposomes in the treatment of infections. Methods in Enzymology, v. 391, p. 12281260, 2005.

BARBOSA, C. I. O. G. N. Novas formas farmacêuticas para uso veterinário, 2010. Disponível em: <http://hdl.handle.net/10284/1611> Acesso em: abr. 2013.

BARROS, M. B. P. Estudo da dinâmica folicular no protocolo de nove dias de sincronização de cios com o uso de um novo dispositivo de liberação sustentada de progesterona, associado ou não ao eCG, em ovelhas Santa Inês. São Paulo: USP, 2010. 65p. Dissertação (Mestrado de Reprodução Animal). Faculdade de Medicina Veterinária e Zootecnia da Universidade de São Paulo, 2010. 
BOETTNER, W. A.; AGUIAR, A. J.; CARDINAL, J. R.; CURTISS, A. C.; RANADE, G. R.; RICHARDS, J. A.; SOKOL, W. F. The morantel sustained release trilaminate: a device for the controlled ruminal delivery of morantel to cattle. Journal of Controlled Release, v. 8, n. 1, p. 23-30, 1988.

BOWERSOCK, T. L.; MARTIN, S. Vaccine delivery to animals. Advanced Drug Delivery Reviews, v. 38, n. 2, p. 167-194, 1999.

BRANDÃO, H. M.; GERN, J. C.; VICENTINI, N. M. et al. Nanotecnologia: a próxima revolução na agropecuária. Revista do Conselho Federal de Medicina Veterinária. XVII, v. 53, p .61-67, 2011.

BRAYDEN, D. J.; OUDOT, E. J. M.; BAIRD, A. W. Drug Delivery Systems in Domestic Animal Species. In: CUNNINGHAM, F.; ELLIOTT, J.; LEES, P. (eds.). Comparative and Veterinary Pharmacology, London: Springer, 2010. P. 79-112.

BULL, M. S.; SWINDALE, S.; OVEREND, D.; et al. Supression of Boophilus microplus populations with fluazuron; an acarine growth regulator. Australian Veterinary Journal, $v$. 74, n. 6, p. 468-470, 1996.

BUNT, C. R.; RATHBONE, M. J.; BURGGRAAF, S.; OGLE, C. R.; BURKE, C. R. Elevation of plasma progesterone levels in cattle using a poly (caprolactone) and cyclodextrin intravaginal insert containing progesterone. Proceedings of the $\mathbf{2 6}$ st International Symposium on Controlled Release of Bioactive Materials, p. 1172-1173, 1999.

BURNS, P. J.; TICET, R.; MASON, D. W. et al. Control of estrus and ovulation in mares using progesterone and estradiol biodegradable microspheres in a multicenter clinical trial, Proceedings of the $\mathbf{2 1}$ st International Symposium on Controlled Release of Bioactive Materials, p.86-88, 1994.

CARDINAL, J. R. Controlled drug delivery: veterinary applications. Journal of Controlled Release, n. 2, p. 393-403, 1985.

CARDINAL, J. R.; WITCHEY-LAKSHMANAN, L. Drug delivery in veterinary medicine. In: KYDONIEUS, A. (ed.). Treatise on Controlled Drug Delivery: Fundamentals, Optimization, Application. New York: Marcel Dekker Inc., 1992. P. 465-489.

CERVONI, J. E. Gordura protegida na alimentação de ruminantes. Disponível em: <http://www.limousin.com.br>. Acesso em: 25 maio 2013.

COUVREUR, P.; VAUTHIER, C. Nanotechnology: intelligent design to treat complex disease. Pharmaceutical Research, v. 23, n. 7, p.1417-1450, 2006.

DALEY, M.; WILLIAMS, T.; COYLE, P.; et al. Prevention and treatment of Staphylococcus aureus infections with recombinant cytokines. Cytokine, v. 5, n. 3, p. 276-284, 1993. 
DAMS E. T.; OYEN W. J., BOERMAN O. C.; et al. 99mTc-PEG liposomes for the scinti graphic detection of infection and inflammation: Clinical evaluation. Journal of Nuclear Medicina, $\mathrm{v}$. 41, p.622-630, 2000.

DASH, A. K.; CUDWORTHLI, G. C. Therapeutic applications of implantable drug delivery systems. Journal of Pharmacological and Toxicological Methods, v. 40, n. 1, p. 1-12, 1998.

DOREAU, M.; CHILLIARD, Y. Digestion and metabolism of dietary fat in farm animals. British Journal of Nutrition, n. 78, p. 15-35, 1997.

DROVER, D. R.; ANGST, M. S.; VALLE, M.; RAMASWAMY, B.; NAIDU, S.; STANSKI, D. R.; VEROTTA, D. Input characteristics and bioavailability after administration of immediate and a new extended-release formulation of hydromorphone in healthy volunteers.

Anesthesiology, n. 97 (4), p. 827-836, 2002.

ELANCO ANIMAL HEALTH. Rumesin ${ }^{\circledR}$ (monensin) for pasture cattle. Disponível em: <http://www.elanco.us/products-services/beef/rumensin-p.aspx>. Acesso em: dez. 2013.

FLEURY, J. J.; SQUIRES, E. L.; BETSCHART, R.; GIBSON, J. W.; SULLIVAN, S. A.; TIPTON, A. J.; BURNS, P. J. Evaluation of the SABER ${ }^{\mathrm{TM}}$ delivery system for the controlled release of deslorelin acetate for advancing ovulation in the mare: effects of formulation and dose. Proceedings International Symposium Controlled Release Bioact Mater, n. 25, p. 657-658, 1998.

FREITAS FILHO, J. R.; NASCIMENTO, A.; SILVA, A. C.; LINO, F. R. L. Medicamentos Veterinários: contextualizando o ensino de Química Orgânica. Acta Scientiae, v. 13, n. 2, p. 129-144, jul/dez 2011.

GEHRING, R.; SMITH, G. W. An overview of factors affecting the disposition of intramammary preparations used to treat bovine mastitis. Journal of Veterinary Pharmacology and Therapeutics, v. 29, n. 4, p. 237-241, 2006.

GEORGE, M. H.; COWMAN, G. L.; TATUM, J. D.; et al. Injection site lesions in beef subprimals: incidence, palatability consequences and economic impact. Compendium of Continuing Education for Practising Veterinarians, v. 19, n. 2, p.84-93, 1997.

GONÇALVES, A.; DOMINGUES, J. L. Uso de gordura protegida na dieta de bovinos. Revista Eletrônica Nutritime, v. 4, n. 5, p. 475-486, 2007.

GRACE, N. D.; KNOWLES, S. O.; SINCLAIR, G. R.; et al. Growth response to increasing doses of microencapsulated vitamin B12 and related changes in tissue vitamin B12 concentrations in cobalt-deficient lambs. New Zealand Veterinary Journal, v. 51, n. 2, p.89-92, 2003. 
GROND, S.; RADBRUCH, L.; LEHMANN, K. A. Clinical pharmacokinetics of transdermal opioids: focus on transdermal fentanyl. Clinical Pharmacokinetics, n. 38, p. 59-89, 2000.

GRUET, P.; MAINCENT, P.; BERTHELOT, X.; et al. Bovine mastitis and intramammary drug delivery: review and perspectives. Advanced Drug Delivery Reviews, v. 50, n. 3, p. 245-259, 2001.

HAFEMAN, S.; LONDON, C.; ELMSLIE, R.; et al. Evaluation of liposomal clodronate for treatment of malignant histiocytosis in dogs. Cancer Immunology, Immunotherapy, v. 59, n. 3, p. 441-452, 2010.

HILBERT, A. K.; FRITZSCHE, U.; KISSEL, T. Biodegradable microspheres containing influenza A vaccine: immune response in mice. Vaccine, v. 17, n. 9-10, p. 1065-1073, 1999.

HORWITZ, E.; KAGAN, L.; AVNI-MAGEN, N.; et al. A novel subcutaneous controlled-release amoxicillin degradable implant for extended-interval administration in veterinary medicine. Journal of Veterinary Pharmacology and Therapeutics, v. 34, n. 5, p. 494-498, 2011.

JOHNSON, C. A.; THOMPSON, D. L.; SULLIVAN, S. A.; GIBSON, J. W.; TIPTON, A. J.; SIMON, B. W.; BURNS, P. J. Biodegradable delivery systems for estradiol comparison between poly(DLlactide) microspheres and the SABER delivery system. Proceedings International Symposium Controlled Release Bioact Mater, n. 26, p. 74-75, 1999.

JOSHI, A.; RAJE, J. Sonicated transdermal drug transport. Journal of Controlled Release, $n$. 83, p. 13-22, 2002.

KASTELIC, J. P.; OLSON, W. O.; MARTINEZ, M.; et al. Sincronização do estro em bovinos Hereford-angus com Crestar. Revista Brasileira de Reprodução Animal, v. 21, n. 2, p. 101 103, 1997.

KAUFMANN, A.; BUTCHER, P.; MADEN, K.; et al. Quantitative multi residue method for about 100 veterinary drugs in different meat matrices by sub $2-\mu \mathrm{m}$ particulate high-performance liquid chromatography coupled to time off light mass spectrometry. Journal of Chromatography A, n. 1194, p. 66-79, 2008.

KLEITER, M.; TICHY, A.; WILLMANN, M.; et al. Concomitant liposomal doxorubicin and daily palliative radiotherapy in advanced feline soft tissue sarcomas. Veterinary Radiology and Ultrasound, v. 51, n. 3, p.349-355, 2010.

KLINK, P. R.; FERGUSON, T. H.; MAGRUDER, J. A. Formulation of veterinary dosage forms. In: HARDEE, G. E.; BAGGOT, J. D. (eds.) Development and Formulation of Veterinary Dosage Forms, New York: Marcel Dekker Inc., 1998. Cap. 2, p. 145-229. 
KUMAR, A.; RAHAL, A.; RAGVENDRA, R.; et al. Pharmacokinetics of levofloxacin following intravenous and intramuscular administration in cattle calves. Asian Journal of Animal and Veterinary Advances, v. 7, n. 10, p. 1006-1013, 2012.

LAINESSE, C.; GEHRING, R.; PASLOSKE, K.; et al. Challenges associated with the demonstration of bioequivalence of intramammary products in ruminants. Journal of Veterinary Pharmacology and Therapeutics, v. 35, suplementos 1, p.65-79, 2012.

LANUSSE, C.; LIFSCHITZ, A.; VIRKEL, G.; et al. Comparative plasma disposition kinetics of ivermectin, moxidectin and doramectin in cattle. Journal of Veterinary Pharmacology and Therapeutics, v. 20, n. 2, p. 91-99, 1997.

LAVY, E.; STEINMAN, A.; SOBACK, S. Oral controlled-release formulation in veterinary medicine. Critical Reviews in Therapeutic Drug Carrier Systems, n. 23, p. 165-204, 2006.

LIMA, K. M.; RODRIGUES-JUNIOR, J. M. Poly-DL-lactide-co-glycolide microspheres as a controlled release antigen delivery system. Brazilian Journal of Medical and Biological Research, v. 32, n. 2, p.171-180, 1999.

LÓPEZ-DONAIRE, M. L.; FERNANDEZ-GUTIÉRREZ, M.; PARRA-CÁCERES, J.; et al. A study on partially biodegradable microparticles as carries of active glycolipids. Acta Biomaterialia, v. 6, n. 4, p. 1360-1369, 2010.

LYNN, R. C.; HEPLER, D. I.; KELCH, W. J.; et al. Double-blinded placebo-controlled clinical field trial to evaluate the safety and efficacy of topically applied $1 \%$ diclofenac liposomal cream for the relief of lameness in horses. Veterinary Therapeutics: research in applied veterinary medicine, v. 5, n. 2, p. 128-138, 2004.

LYRA, M. A. M.; SOARES SOBRINHO, J. L.; BRASILEIRO, M. T.; LA ROCA, M. F.; BARRAZA, J. A.; VIANA, O. S.; ROLIM NETO, P. J. Sistemas matriciais hidrofílicos e mucoadesivos para liberação controlada de fármacos. Latin American Journal of Pharmacy, v. 26, n. 5, p. 784793, 2007.

MACKLE, T. R.; DWYER, D. A.; BAUMAN, D. E. Intramammary infusion of Insulin or Long R3 Insulin-like Growth Factor-I did not increase milk protein yield in dairy cows. Journal of Dairy Science, v. 83, n. 8, p.1740-1949, 2000.

MAGNUSSON, B. M.; WALTERS, K. A.; ROBERTS, M. S. Veterinary drug delivery: potential for skin penetration enhancement. Advanced Drug Delivery Reviews, n. 50, p. 205-227, 2001.

MARTIN, C. R.; KOHLI, P. The emerging field of nanotube biotechnology. Nature Reviews Drug Discovery, v. 2, n.1, p. 29-37, 2003. 
MATSCHKE, C.; ISELE, U.; VAN HOOGEVEST, P.; FAHR, A. Sustained release injectables formed in situ and their potential use for veterinary products. Journal of Controlled Release, v. 85, n. 1-3, p. 1-15, 2002.

MEDLICOTT, N. J.; WALDRON, N. A.; FOSTER, T. P. Sustained release veterinary parenteral products. Advanced Drug Delivery Reviews, v. 56, n. 10, p. 1345-1365, 2004.

MILLER, A. J.; OEHLER, D. D.; POUND, M. J. Delivery of ivermectin by injectable microspheres. Journal of Economic Entomology, v. 91, n. 3, p. 655-659, 1998.

MILLER, J. A. Controlled release products for control of ectoparasites of livestock. In: RATHBONE, M. J.; GURNY, R. Controlled Release Veterinary Drug Delivery - biological and pharmaceutical considerations, Amsterdam: Elsevier, 2000. Cap. 8, p. 229-248.

MILLER, J. A.; DAVEY, R. B.; OEHLER, D. D.; et al. The Ivomec SR Bolus for control of Boophilus annulatus (Acari: Ixodidae) on cattle in South Texas. Journal of Economic Entomology, n. 94, p. 1622-1627, 2001.

MILLS, P. C.; CROSS, S. E. Transdermal drug delivery:basic principles for the veterinarian. Veterinary Journal, v. 172, n. 2, p. 218-233, 2006.

PELL, A. N.; WU, S. H.; WELCH, J. G. Design parameters for post ruminal drug delivery systems and rumen-stable products. In: RATHBONE, M. J.; GURNY, R. (eds.). Controlled Release Veterinary Drug Delivery: Biological and Pharmaceutical Considerations. Amsterdam:Elsevier, 2000. P. 83-113.

PEREZ, B.; PRATS, C.; CASTELLS, E.; et al. Determination of cloxacillin in milk and blood of dairy cows by high-performance liquid chromatography. Journal of Chromatography B: Biomedical Sciences and Applications, v .698,n. 1-2, p.155-160, 1997.

PEREZ, C.; CASTELLANOS, I. J.; COSTANTINO, H. R.; et al. Recent trends in stabilizing protein structure upon encapsulation and release from bioerodible polymers. Journal of Pharmacy and Pharmacology, v. 54, n. 3, p.301-313, 2002.

PFIZER SAÚDE ANIMAL. Formoped ${ }^{\circledR}$. Disponível em: <http://www2.pfizersaudeanimal.com.br/ovinos_caprinos/bulas/Formoped.pdf > Acesso em: jun. 2013.

PINTO, C.; SANTOS, V. M.; DINIS, J.; PELETEIRO, M. C.; FITZGERALD, J. M.; HAWKES, A. D.; SMITH, B. L. Pithomycotoxicosis (facial eczema) in ruminants in the Azores, Portugal. Veterinary Record, n. 157, p. 805-810, 2005.

PRESTON, R. L. Hormone containing growth promoting implants in farmed livestock. Advanced Drug Delivery Reviews, v. 38, n. 2, p. 123-138, 1999. 
RATHBONE, M. J.; WITCHEY-LAKSHMANAN, L.; CIFTCI, K. Veterinary applications. IN: MATHIOWITZ, E. (ed.) Encyclopedia of Controlled Drug Delivery. New York: Wiley, 1999. P. 1007-1037.

RATHBONE, M. J.; MARTINEZ, M. N. Modified release drug delivery in veterinary medicine. Drug Discovery Today, n. 7, p .823-829, 2002.

RATHBONE, M. J.; BRAYDEN, D. Controlled release drug delivery in farmed animals: Commercial challenges and academic opportunities. Current Drug Delivery, n. 6, p. 383-390, 2009.

REEVES, P. T. Residues of veterinary drugs at injection sites. Journal of Veterinary Pharmacology and Therapeutics, v. 30, n. 1, p. 1-17, 2007.

REVERCHON, E. Supercritical antisolvent precipitation of micro-and nano-particles. The Journal of Supercritical Fluids, v .15, n. 1, p. 1-21, 1999.

ROBERTS, M. S.; CROSS, S. E.; PELLETT, M. A. Skin transport. In: WALTERS, K. A. (ed.), Dermatological and Transdermal Formulations. New York: Marcel Dekker, 2002. P. 89-196.

ROCHA, D. C.; BESKOW, A.; PIMENTEL, C. M. M. et al. Níveis séricos de progesterona em vacas ovariectomizadas tratadas com MAD4 ${ }^{\circledR}$ com diferentes concentrações e vias de administração. Acta Scientiae Veterinariae, v. 39, n. 3, publicação 974, p.1-6, 2011.

ROTHEN-WEINHOLD, A.; GURNY, R.; DAHN, M. Formulation and technology aspects of controlled drug delivery in animals. Pharmaceutical Science \& Technology Today, v. 3, n. 7, p. 222-231, 2000.

RUBIANES, E. Nociones básicas de fisiologia reproductiva em cabras y ovejas. IN: Simpósio sobre controle farmacológico do ciclo estral em ruminantes. São Paulo - SP Anais. São Paulo: Fundação da Faculdade de Medicina Veterinária e Zootecnia, USP. 2000. P.255-282.

SATO, K.; UENO, S. Polymorphism in fats and oils. In: SHAHIDI, F. (ed.). Bailey's Industrial oil and fat products. New York: John Wiley, 2005. Cap. 3, p. 77-120.

SCHAFFAZICK, S. R.; GUTERRES, S. S.; FREITAS, L. L. et al. Physicochemical characterization and stability of the polymeric nanoparticle systems for drug administration. Química Nova., v. 26, n. 5, p. 726-737, 2003.

SCHAFFAZICK, S. R.; POHLMANN, A. R.; DALLA-COSTA, T.; et al. Freeze drying polymeric colloidal suspensions: nanocapsules, nanospheres and nanodispersion: a comparative study. European Journal of Pharmaceutics and Biopharmaceutics, v. 56, n. 3, p. 501-505, 2003. 
SHAHANI, K.; SWAMINATHAN, S. K.; FREEMAN, D.; et al. Injectable sustained release microparticles of curcumin: a new concept for cancer chemoprevention. Cancer Research, v. 70, n. June 1, p. 4443-4452, 2010.

SMITH, D. R.; GROTELUESCHEN, D. M.; GRIFFIN, D. D. Proper Injection Procedures for Cattle. Historical Materials from University of Nebraska-Lincoln Extension paper 349, Nebraska, 1998. Disponível em: http://digitalcommons.unl.edu/extensionhist/349_Acesso em: março de 2014.

SORDILLO, L. M; SHAFER-WEAVER, K.; DEROSA, D. Immunobiology of the mammary gland. Journal of Dairy Science, v. 80, n. 8, p.1851-1865, 1997.

SPINOSA, H. S.; GORNIAK, S. I.; BERNARDI, M. M. Farmacologia Aplicada à Medicina Veterinária. 2. ed. Rio de Janeiro: Guanabara Koogan, 1999. 946p.

STROMBERG, B. E.; AVERBECK, G. A.; ANDERSON, J. E.; et al. Comparison of the persistent efficacy of the injectable and pour-on formulations of doramectin against artificially-induced infection with Dictyocaulus viviparous in cattle. Veterinary Parasitology, v. 87, n. 1, p. 45-50, 1999.

TEICHMANN, A.; HEUSCHKEL, S.; JACOBI, U.; PRESSE, G.; NEUBERT, R. H. H.; STERRY, W.; LADEMANN, J. Comparison of stratum corneum penetration and localization of a lipophilic model drug applied in an o/w microemulsion and an amphiphilic cream. European Journal of Pharmaceutics and Biopharmaceutics, v. 67, p. 699-706, 2007.

TREGASKES, L. D.; BROADBENT, P. J.; DOLMAN, D. F.; et al. Evaluation of Crestar, a synthetic progestogen regime, for synchronizing oestrus in maiden heifers used asrecipients of embryo transfers. Veterinary Record, v.134, n. 4, p.92-94, 1994.

USUI, T.; KONNAI, S.; TARIMA, S.; et al. Protective effects of vaccination with bovine leukemia virus (BLV) Tax DNA against BLV infection in sheep. The Journal of Veterinary Medical Science, v. 65, n. 11, p. 1201-1205, 2003.

VALENTIM, R. C.; CORREIA, T. M.; AZEVEDO J. M. Utilização de implantes de melatonina em ovinos. Albéitar Portuguesa, v. 2, n. 6, p. 18-22, 2006.

VAN DEN BOGAARD, A. E.; STOBBERINGH, E. E. Epidemiology of resistance to antibiotics. Links between animals and humans. International Journal of Antimicrobial Agents, n. 14, p. 327-35, 2000.

VANDAMME, Th. F.; ELLIS, K. J. Issues and Challenges in Developing Ruminal Drug Delivery Systems, Advanced Drug Delivery Reviews, v. 56, n. 10, p. 1416-1436, 2004. 
WHEATON, J. E; CARLSON, K. M.; WINDELS, H. F.; JOHNSTON, L. J. CIDR: a new progesteronereleasing intravaginal device for induction of estrus and cycle control in sheep and goats.

Animal Reproduction Science, n. 33, p. 127-141, 1993.

WINZENBURG, G.; SCHIMIDT, C.; FUCHS, S. et al. Biodegradable polymers and their potential use in parenteral veterinary drug delivery systems. Advanced Drug Delivery Reviews, v. 56, n. 10, p. 1453-1466, 2004.

WISSING, S. A.; KAYSERB, O.; MÜLLER, R. H. Solid lipid nanoparticles for parenteral drug delivery. Advanced Drug Delivery Reviews, v. 56, n. 9, p. 1257-7122, 2004.

WRIGHT, J. Pumps/osmotic-ruminal osmotic bolus. In: MATHIOWITZ, E. (ed.). Encyclopedia of Controlled Drug Delivery. Ann Arbor: Wiley, 1999. P. 915-920.

Autor para correspondência: Rafael da Fonseca Prietsch. Universidade Federal de Pelotas - Campus Universitário Capão do Leão. CEP 96010900 - caixa Postal 354; Pelotas (RS)Brasil. rafaelprietsch@hotmail.com 